MATHEMATICS OF COMPUTATION

Volume 70, Number 233, Pages 431-447

S $0025-5718(00) 01280-1$

Article electronically published on June 12, 2000

\title{
EXPLICIT UPPER BOUNDS FOR EXPONENTIAL SUMS OVER PRIMES
}

\author{
HEDI DABOUSSI AND JOËL RIVAT
}

Dedicated to the memory of Chen Jing Run

Abstract. We give explicit upper bounds for linear trigonometric sums over primes.

\section{INTRODUCTION}

In 1937 I.M. Vinogradov 12 proved that every sufficiently large odd number is the sum of three prime numbers. Later Chen and Wang 2 gave a lower bound for the result of Vinogradov, which is very large, around $10^{43000}$. The method used is the Hardy-Littlewood circle method, and the following sums play an important role in the proof:

$$
\sum_{p \leq x} \mathrm{e}(\alpha p), \quad S(x, \alpha)=\sum_{n \leq x} \Lambda(n) \mathrm{e}(n \alpha)
$$

where $\Lambda$ is the function of Von Mangoldt and $\mathrm{e}(\alpha)=e^{2 i \pi \alpha}$.

In [1] Chen proved that if $\alpha=\frac{a}{q}+\frac{\beta}{q^{2}},|\beta| \leq 1, q \leq x$, then

$$
\left|\sum_{p \leq x} \mathrm{e}(\alpha p)\right| \leq 1.2 x(\log x)^{3 / 4} \log \log x\left(\sqrt{\frac{5}{q}+\frac{q \log q}{x}}+\sqrt{\log q} \exp -\frac{1}{2} \sqrt{\log x}\right) .
$$

More recently in 3 . Chen and Wang proved that

$$
|S(x, \alpha)| \leq 0.177 \frac{x}{\sqrt{q}}(\log x)^{3}+3.8 x^{4 / 5}(\log x)^{2.2}+0.08 \sqrt{x q}(\log x)^{3.5} .
$$

Our purpose is to improve on these two estimates. By a classical elementary transformation it suffices to consider $S(x, \alpha)$.

In order to estimate this sum, a useful identity has been proved by R.C. Vaughan 11]. Recently Daboussi [4] gave another identity, which has the advantage of involving nice coefficients. This permits us to give a new explicit upper bound for $|S(x, \alpha)|$.

In this paper we will need sharp versions of some classical inequalities which have their own independent interest. We will prove

Received by the editor November 3, 1998.

2000 Mathematics Subject Classification. Primary 11L07, 11L20.

Key words and phrases. Prime numbers, exponential sums, sieves.

(C)2000 American Mathematical Society 
TABLE 1. Upper bounds for $x^{-1}|S(x, \alpha)|$ when $q=(\log x)^{3}$

\begin{tabular}{||l|l||}
\hline \multicolumn{1}{|c|}{$x$} & $x^{-1}|S(x, \alpha)|$ \\
\hline $10^{200}$ & 0.385 \\
$10^{300}$ & 0.293 \\
$10^{400}$ & 0.241 \\
$10^{500}$ & 0.207 \\
$10^{1000}$ & 0.129 \\
$10^{2000}$ & 0.080 \\
$10^{5000}$ & 0.042 \\
$10^{10000}$ & 0.026 \\
$10^{20000}$ & 0.016 \\
$10^{43000}$ & 0.010 \\
\hline
\end{tabular}

Theorem 1. For $\alpha=\frac{a}{q}+\frac{\beta}{q^{2}},|\beta| \leq 1, q \leq x$, we have

$$
\begin{aligned}
|S(x, \alpha)| \leq & 14.86 \sqrt{\log \log x+0.5} x(\log x)^{3 / 4} \sqrt{\frac{1}{q}+\frac{q \log 4 q}{\pi x}} \\
& +6.45 \sqrt{\log \log x+0.5} x(\log x)^{5 / 4} \exp \left(-\frac{1}{2} \sqrt{\log x}\right) .
\end{aligned}
$$

From this theorem we can compute numerical upper bounds for $x^{-1}|S(x, \alpha)|$ (cf. Table 1) with the choice $q=(\log x)^{3}$ for which the result of Chen and Wang is not even as good as the trivial upper bound.

Definitions and notations. For $x$ real we will denote by $\lfloor x\rfloor$ the greatest integer $\leq x,\{x\}$ the fractional part of $x,\lceil x\rceil$ the smallest integer $\geq x,\|x\|$ the distance from $x$ to the nearest integer, $\lfloor x\rceil$ the smallest integer $n$ such that $|x-n| \leq 1 / 2$ ( $n$ is unique if $\{x\} \neq 1 / 2$ ). The letter $p$ denotes always a prime number, $\pi(x)$ denotes the number of primes $\leq x$. We denote by $\mu$ and $\varphi$ the Möbius and Euler functions, respectively. The functions $\Omega(n)$ and $\omega(n)$ count the number of prime factors of $n$, respectively, with and without multiplicity. We define the functions $u_{z}$ and $v_{z}$ by $u_{z}(m)=1$ if $(\forall p, p \mid m \Rightarrow p>z)$ and $u_{z}(m)=0$ otherwise, and $v_{z}(m)=1$ if $(\forall p, p \mid m \Rightarrow p \leq z)$ and $v_{z}(m)=0$ otherwise.

\section{VinOGRADOV TYPE LEMMAS}

In [1] Chen improved Vinogradov Lemmas 8a and 8b [13]. In this section, we further improve the results of Chen.

Lemma 1. Let $x \in \mathbb{R}, \alpha=\frac{a}{q}+\frac{\beta}{q^{2}},|\beta| \leq 1,(a, q)=1, U>0$. We then have

$$
\sum_{x<n \leq x+q} \min \left(U, \frac{1}{|\sin (\pi \alpha n)|}\right) \leq 2 U+\frac{2}{\pi} q \log 4 q .
$$

Remark 1. This is the analog of Vinogradov Lemma 8a. Chen obtained $5 U+q \log q$. The factor 2 instead of 5 is obtained by using $t=\lfloor t\rceil+\delta$ with $|\delta| \leq 1 / 2$ which is more precise than the classical $t=\lfloor t\rfloor+\{t\}$. The factor $2 / \pi$ has been obtained by dealing directly with $(\sin t)^{-1}$ without using the classical inequality $\sin t \geq 2 t / \pi$ for 
$0 \leq t \leq \pi / 2$. Indeed we simply used the fact that $(\log \tan (t / 2))^{\prime}=(\sin t)^{-1}$. We acknowledge the referee's improvement of this lemma (see below).

Proof. The result is trivial for $q \leq 2$. We therefore suppose $q \geq 3$.

Let $m_{0}=\lfloor x\rfloor+\lfloor(q+1) / 2\rfloor$. We have

$$
\sum_{x<n \leq x+q} \min \left(U, \frac{1}{|\sin (\pi \alpha n)|}\right)=\sum_{-q / 2<m \leq q / 2} \min \left(U, \frac{1}{\left|\sin \left(\pi \alpha\left(m_{0}+m\right)\right)\right|}\right) .
$$

Now writing

$$
b=\left\lfloor a m_{0}+\frac{\beta m_{0}}{q}\right\rceil \text { and } \quad b+\delta=a m_{0}+\frac{\beta m_{0}}{q}
$$

(hence $|\delta| \leq 1 / 2$ ), we obtain

$$
\alpha\left(m_{0}+m\right)=\frac{1}{q}\left(a m+a m_{0}+\frac{\beta m_{0}}{q}+\frac{\beta m}{q}\right)=\frac{1}{q}(a m+b)+\frac{1}{q}\left(\delta+\frac{\beta m}{q}\right) .
$$

When $m$ runs through the integers in the interval $-q / 2<m \leq q / 2, a m+b$ runs through a complete set of residue classes modulo $q$. We introduce $r$ such that $a m+b \equiv r \bmod q$ and $-q / 2<r \leq q / 2$. Using $\left|\delta+\frac{\beta m}{q}\right| \leq 1$, we get for $|r| \geq 2$

$$
\min \left(U, \frac{1}{\left|\sin \left(\pi \alpha\left(m_{0}+m\right)\right)\right|}\right) \leq \sin \left(\frac{\pi}{q}(|r|-1)\right) .
$$

For $r= \pm 1$, the referee observed that

$$
\left\|\alpha\left(m_{0}+m\right)\right\|=\left\|\frac{f(r)}{q}\right\|
$$

where $f(r)=r+\delta+\theta(r)$, with $|\theta(r)| \leq \frac{1}{2}$. It follows that $f(1)-f(-1) \geq 1$ so that

$$
\max \left\{\left\|\frac{f(1)}{q}\right\|,\left\|\frac{f(-1)}{q}\right\|\right\} \geq \frac{1}{2 q} .
$$

Thus one of the two terms for $r= \pm 1$ can be bounded by $|\sin (\pi / 2 q)|^{-1}$. Hence we obtain

$$
\sum_{x<n \leq x+q} \min \left(U, \frac{1}{|\sin (\pi \alpha n)|}\right) \leq 2 U+\frac{1}{\sin \left(\frac{\pi}{2 q}\right)}+2 \sum_{2 \leq r \leq q / 2} \frac{1}{\sin \left(\frac{\pi}{q}(r-1)\right)}
$$

(the sum on the right hand side is empty for $q=3$ ).

Using the convexity of the function $t \longmapsto 1 / \sin (\pi t / q)$ for $0<t \leq q / 2$, we obtain

$$
\sum_{1 \leq r \leq \frac{q}{2}-1} \frac{1}{\sin \frac{\pi}{q} r} \leq \int_{\frac{1}{2}}^{\frac{q-1}{2}} \frac{d t}{\sin \frac{\pi t}{q}} \leq \frac{q}{\pi} \log \cot \frac{\pi}{4 q} \leq \frac{q}{\pi} \log \frac{4 q}{\pi},
$$

and we have for $q \geq 3$

$$
\frac{1}{\sin \left(\frac{\pi}{2 q}\right)}+\frac{2 q}{\pi} \log \frac{4 q}{\pi} \leq \frac{2 q}{\pi} \log 4 q
$$

which completes the proof of Lemma 1.

Lemma 2. Let $N \geq 1, \alpha=\frac{a}{q}+\frac{\beta}{q^{2}},|\beta| \leq 1,(a, q)=1, U>0$. We then have

$$
\sum_{1 \leq n \leq N} \min \left(U, \frac{1}{|\sin (\pi \alpha n)|}\right) \leq\left\lceil\frac{N}{q}\right\rceil\left(2 U+\frac{2}{\pi} q \log 4 q\right) .
$$


Proof. We divide the interval $1 \leq n \leq N$ into subintervals $k q+1 \leq n \leq(k+1) q$, for which we apply Lemma 1 . There are at most $\left\lceil\frac{N}{q}\right\rceil$ such subintervals.

Lemma 3. Let $N \geq 1, \alpha=\frac{a}{q}+\frac{\beta}{q^{2}},|\beta| \leq 1,(a, q)=1, x>0$. We then have

$$
\sum_{1 \leq n \leq N} \min \left(\frac{x}{n}, \frac{1}{|\sin (\pi \alpha n)|}\right) \leq 2 \frac{x}{q} \log \left(\frac{8 N}{q}+4\right)+\frac{2}{\pi} N \log 4 q+\frac{3}{\pi} q \log 5 q .
$$

Remark 2. This is the analog of Vinogradov Lemma $8 \mathrm{~b}$.

Proof. We can assume without loss of generality that $N$ is an integer.

Using the convexity of $t \longmapsto \frac{1}{t}$ for $t>0$, we obtain for $N \geq 1$

$$
\sum_{1 \leq n \leq N} \frac{1}{n} \leq \int_{\frac{1}{2}}^{N+\frac{1}{2}} \frac{d t}{t}=\log (2 N+1) .
$$

This proves the result for $q \leq 2$. We can now suppose $q \geq 3$.

Writing $K=\left\lceil\frac{N}{q}-\frac{1}{2}\right\rceil$, we have $K \geq \frac{N}{q}-\frac{1}{2}$ and $K q+\frac{q}{2} \geq N$. Hence

$$
\sum_{1 \leq n \leq N} \min \left(\frac{x}{n}, \frac{1}{|\sin (\pi \alpha n)|}\right) \leq \sum_{k=0}^{K} S_{k},
$$

where

$$
S_{0}=\sum_{1 \leq n \leq q / 2} \min \left(\frac{x}{n}, \frac{1}{|\sin (\pi \alpha n)|}\right)
$$

and for $k \geq 1$

$$
S_{k}=\sum_{k q-q / 2<n \leq k q+q / 2} \min \left(\frac{x}{n}, \frac{1}{|\sin (\pi \alpha n)|}\right) .
$$

For $1 \leq n \leq q / 2$, we have an $\not \equiv 0 \bmod q$ and $\alpha n=\frac{a n}{q}+\frac{1}{q} \frac{\beta n}{q}$ with $\left|\frac{\beta n}{q}\right| \leq \frac{1}{2}$. Hence for $a n \equiv r \bmod q$ with $-q / 2 \leq r \leq q / 2$ and $r \neq 0$, we have

$$
|\sin (\pi \alpha n)| \geq\left|\sin \left(\frac{\pi}{q}\left(|r|-\frac{1}{2}\right)\right)\right|
$$

and

$$
\begin{aligned}
S_{0} & \leq 2 \sum_{1 \leq r \leq q / 2}\left|\sin \left(\frac{\pi}{q}\left(r-\frac{1}{2}\right)\right)\right|^{-1} \\
& \leq 2\left|\sin \left(\frac{\pi}{2 q}\right)\right|^{-1}+2 \int_{\frac{3}{2}}^{\frac{q+1}{2}} \frac{d t}{\sin \left(\frac{\pi}{q}\left(t-\frac{1}{2}\right)\right)} \\
& \leq \frac{2}{\sin \left(\frac{\pi}{2 q}\right)}+\frac{2 q}{\pi} \log \cot \left(\frac{\pi}{2 q}\right) \\
& \leq \frac{2 q}{\pi}\left(2 \frac{\frac{\pi}{2 q}}{\sin \left(\frac{\pi}{2 q}\right)}+\log \left(\frac{2 q}{\pi}\right)\right) \\
& \leq \frac{2 q}{\pi} \log 5 q \quad \text { for } q \geq 4 .
\end{aligned}
$$


For $q=3$ we also have $S_{0} \leq 2\left|\sin \frac{\pi}{2 q}\right|^{-1}=4 \leq \frac{2 q}{\pi} \log 5 q$. This proves the result for $K<1$, so from now we assume that $K \geq 1$.

By Lemma 1 we have

$$
\begin{aligned}
\sum_{1 \leq k \leq K} S_{k} & \leq \sum_{1 \leq k \leq K} \sum_{k q-q / 2<n \leq k q+q / 2} \min \left(\frac{x}{q\left(k-\frac{1}{2}\right)}, \frac{1}{|\sin (\pi \alpha n)|}\right) \\
& \leq \sum_{1 \leq k \leq K}\left(2 \frac{x}{q\left(k-\frac{1}{2}\right)}+\frac{2}{\pi} q \log 4 q\right) \\
& \leq \frac{2}{\pi} K q \log 4 q+\frac{2 x}{q} \sum_{1 \leq k \leq K} \frac{1}{\left(k-\frac{1}{2}\right)} .
\end{aligned}
$$

Hence,

$$
\begin{aligned}
\sum_{1 \leq k \leq K} S_{k} & \leq \frac{2}{\pi} K q \log 4 q+\frac{2 x}{q}\left(2+\int_{\frac{3}{2}}^{K+\frac{1}{2}} \frac{d t}{\left(t-\frac{1}{2}\right)}\right) \\
& \leq \frac{2}{\pi} K q \log 4 q+\frac{2 x}{q}(2+\log K) \\
& \leq \frac{2}{\pi}\left(\frac{N}{q}+\frac{1}{2}\right) q \log 4 q+\frac{2 x}{q} \log \left(e^{2} K\right) \\
& \leq \frac{2}{\pi} N \log 4 q+\frac{q}{\pi} \log 4 q+\frac{2 x}{q} \log \left(e^{2} K\right)
\end{aligned}
$$

Finally,

$$
\begin{aligned}
\sum_{1 \leq n \leq N} \min & \left(\frac{x}{n}, \frac{1}{|\sin (\pi \alpha n)|}\right) \\
& \leq \frac{2}{\pi} N \log 4 q+\frac{2 q}{\pi}\left(\log q+\log 5+\frac{\log q}{2}+\frac{\log 4}{2}\right)+\frac{2 x}{q} \log \left(e^{2} K\right) \\
& \leq \frac{2}{\pi} N \log 4 q+\frac{3 q}{\pi}\left(\log q+\frac{2 \log 5}{3}+\frac{\log 4}{3}\right)+\frac{2 x}{q} \log \left(e^{2} K\right) \\
& \leq \frac{2}{\pi} N \log 4 q+\frac{3 q}{\pi} \log 5 q+\frac{2 x}{q} \log \left(e^{2} K\right) \\
& \leq \frac{2}{\pi} N \log 4 q+\frac{3 q}{\pi} \log 5 q+\frac{2 x}{q} \log \left(\frac{8 N}{q}+4\right),
\end{aligned}
$$

which completes the proof.

\section{RANKIN'S METHOD}

Elliott [6, pages 81-83] has given an effective version of Rankin's method. In this section we generalize and improve his results numerically.

Lemma 4. Let $z \geq 2, f$ a multiplicative function with $f \geq 0$, and

$$
S=\sum_{p \leq z} \frac{f(p)}{1+f(p)} \log p .
$$

We assume $S>0$ and write $K(t)=\log t-1+\frac{1}{t}$ for $t \geq 1$. 
For any $y$ with $\log y \geq S$ we have

$$
\begin{aligned}
& \sum_{n>y} v_{z}(n) \mu^{2}(n) f(n) \leq\left(\prod_{p \leq z}(1+f(p))\right) \exp \left(-\frac{\log y}{\log z} K\left(\frac{\log y}{S}\right)\right), \\
& \sum_{n \leq y} v_{z}(n) \mu^{2}(n) f(n) \geq\left(\prod_{p \leq z}(1+f(p))\right)\left(1-\exp \left(-\frac{\log y}{\log z} K\left(\frac{\log y}{S}\right)\right)\right) .
\end{aligned}
$$

In particular for any $y$ with $\log y \geq 7 S$ we have

$$
\begin{aligned}
& \sum_{n>y} v_{z}(n) \mu^{2}(n) f(n) \leq\left(\prod_{p \leq z}(1+f(p))\right) \exp \left(-\frac{\log y}{\log z}\right), \\
& \sum_{n \leq y} v_{z}(n) \mu^{2}(n) f(n) \geq\left(\prod_{p \leq z}(1+f(p))\right)\left(1-\exp \left(-\frac{\log y}{\log z}\right)\right) .
\end{aligned}
$$

Proof. The special case for $\log y \geq 7 S$ is a direct consequence of the general case $\log y \geq S$, as for all $t \geq 7$ we have $K(t) \geq K(7) \geq 1$.

We note that

$$
\sum_{n \leq y} v_{z}(n) \mu^{2}(n) f(n)+\sum_{n>y} v_{z}(n) \mu^{2}(n) f(n)=\prod_{p \leq z}(1+f(p))
$$

which shows that the required lower bound for the first sum will follow from the required upper bound for the second sum.

For all $\eta \geq 0$ we have

$$
\begin{aligned}
\sum_{n>y} v_{z}(n) \mu^{2}(n) f(n) & \leq \sum_{n=1}^{\infty} v_{z}(n) \mu^{2}(n) f(n)\left(\frac{n}{y}\right)^{\eta} \\
& \leq y^{-\eta} \prod_{p \leq z}\left(1+f(p) p^{\eta}\right)
\end{aligned}
$$

Now

$$
\prod_{p \leq z}\left(1+f(p) p^{\eta}\right)=\left(\prod_{p \leq z}(1+f(p))\right)\left(\prod_{p \leq z}\left(1+\frac{f(p)}{1+f(p)}\left(p^{\eta}-1\right)\right)\right)
$$

Using $\log (1+u) \leq u$ for $u \geq 0$ we get

$$
\prod_{p \leq z}\left(1+\frac{f(p)}{1+f(p)}\left(p^{\eta}-1\right)\right) \leq \exp \left(\sum_{p \leq z} \frac{f(p)}{1+f(p)}\left(p^{\eta}-1\right)\right)
$$




$$
\begin{aligned}
\sum_{p \leq z} \frac{f(p)}{1+f(p)}\left(p^{\eta}-1\right) & \leq \sum_{p \leq z} \frac{f(p)}{1+f(p)}(\exp (\eta \log p)-1) \\
& \leq \sum_{p \leq z} \frac{f(p)}{1+f(p)} \sum_{k=1}^{\infty} \frac{(\eta \log p)^{k}}{k !} \\
& \leq \sum_{k=1}^{\infty} \frac{\eta^{k}(\log z)^{k-1}}{k !} \sum_{p \leq z} \frac{f(p)}{1+f(p)} \log p \\
& \leq \frac{S}{\log z} \sum_{k=1}^{\infty} \frac{\eta^{k}(\log z)^{k}}{k !} \\
& \leq \frac{S}{\log z}(\exp (\eta \log z)-1) .
\end{aligned}
$$

Writing $\nu=\eta \log z$ we get

$$
y^{-\eta} \prod_{p \leq z}\left(1+\frac{f(p)}{1+f(p)}\left(p^{\eta}-1\right)\right) \leq \exp \left(\frac{S}{\log z}\left(\exp (\nu)-1-\nu \frac{\log y}{S}\right)\right) .
$$

The last inequality is valid for any $\nu \geq 0$, in particular for $\nu=\log \left(\frac{\log y}{S}\right)$. Hence

$$
\begin{aligned}
& \sum_{n>y} v_{z}(n) \mu^{2}(n) f(n) \\
& \leq\left(\prod_{p \leq z}(1+f(p))\right) \exp \left(\frac{S}{\log z}\left(\frac{\log y}{S}-1-\frac{\log y}{S} \log \left(\frac{\log y}{S}\right)\right)\right) \\
& \quad \leq\left(\prod_{p \leq z}(1+f(p))\right) \exp \left(-\frac{\log y}{\log z} K\left(\frac{\log y}{S}\right)\right) .
\end{aligned}
$$

\section{EfFective inequalities}

Lemma 5. For all $x>1$ we have

$$
\pi(2 x)-\pi(x)<\frac{x}{\log x}
$$

Proof. P. Dusart [5] improved some results of [9] and proved for $x \geq 60184$ that

$$
\frac{x}{\log (x)-1}<\pi(x)<\frac{x}{\log (x)-1.1} .
$$

This implies for $x \geq 60184$ that

$$
\pi(2 x)-\pi(x) \leq \frac{2 x}{\log x-0.41}-\frac{x}{\log x-1} \leq \frac{x}{\log x}\left(1-\frac{0.016}{\log x}\right)<\frac{x}{\log x}
$$

using the inequalities $1+u<\frac{1}{1-u}<1+\frac{6}{5} u$ (valid for $0<u<1 / 6$ ). The result can be easily extented for all $x>1$ by computer evidence.

Remark 3. We note that the result of this lemma is sharp for $x=113 / 2$ for which

$$
\pi(113)-\pi(113 / 2)=14<\frac{113 / 2}{\log (113 / 2)}=14.0051 \ldots
$$


Lemma 6. For $z \geq 2$ we have

$$
\sum_{q \leq z}\left(1+\frac{q}{z}\right)^{-1} \frac{\mu^{2}(q)}{\varphi(q)} \geq \log z
$$

Proof. By Lemma 8 of Montgomery-Vaughan [7] we have for $z \geq 100$

$$
\sum_{q \leq z}\left(1+\frac{q}{z}\right)^{-1} \frac{\mu^{2}(q)}{\varphi(q)} \geq \log z+0.361
$$

and the result follows by a direct computation for $2 \leq z<100$.

Lemma 7. For $2 \leq z \leq x$ we have

$$
\sum_{x<m \leq 2 x} u_{z}(m) \leq \frac{x}{\log z} .
$$

Proof. Suppose first that $\sqrt{2 x} \leq z \leq x$. For $x<m \leq 2 x$, we have $u_{z}(m)=1$ if and only if $m$ is prime. Using Lemma 5 we obtain

$$
\sum_{x<m \leq 2 x} u_{z}(m)=\pi(2 x)-\pi(x) \leq \frac{x}{\log x} \leq \frac{x}{\log z} .
$$

Hence we can suppose $z<\sqrt{2 x}$.

By Corollary 1 of Montgomery-Vaughan [7] we have for any positive number $z$,

$$
\sum_{x<m \leq 2 x} u_{z}(m) \leq x\left(\sum_{q \leq z}\left(1+\frac{3 q z}{2 x}\right)^{-1} \frac{\mu^{2}(q)}{\varphi(q)}\right)^{-1},
$$

and using Lemma 6 we obtain for $z \leq \sqrt{\frac{2}{3} x}$

$$
\sum_{x<m \leq 2 x} u_{z}(m) \leq x\left(\sum_{q \leq z}\left(1+\frac{q}{z}\right)^{-1} \frac{\mu^{2}(q)}{\varphi(q)}\right)^{-1} \leq \frac{x}{\log z} .
$$

Thus we can suppose $\sqrt{\frac{2}{3} x}<z<\sqrt{2 x}$.

For $x>15$ we have $(2 x)^{1 / 3}<\sqrt{\frac{2}{3} x}<z<\sqrt{2 x}$ and

$$
\sum_{x<m \leq 2 x} u_{z}(m)=\pi(2 x)-\pi(x)+\pi_{2}(2 x, z)-\pi_{2}(x, z),
$$

where

$$
\pi_{2}(x, z)=\#\{n \leq x, \Omega(n)=2, p \mid n \Longrightarrow p>z\} .
$$

We have using Lemma 5

$$
\pi_{2}(2 x, z)-\pi_{2}(x, z) \leq \sum_{z<p \leq \sqrt{2 x}}\left(\pi\left(\frac{2 x}{p}\right)-\pi\left(\frac{x}{p}\right)\right) \leq \sum_{z<p \leq \sqrt{2 x}} \frac{x / p}{\log (x / p)} .
$$

For $x>15$ we have $x / z>e$ and the function $t \longmapsto t / \log t$ is increasing for $t>e$. Hence

$$
\pi_{2}(2 x, z)-\pi_{2}(x, z) \leq \frac{x / z}{\log (x / z)}(\pi(\sqrt{2 x})-\pi(z)) \leq \frac{x / z}{\log (x / z)}(\pi(2 z)-\pi(z)),
$$


and using Lemma 5 we obtain

$$
\pi_{2}(2 x, z)-\pi_{2}(x, z) \leq \frac{x}{\log (x / z) \log z} .
$$

Therefore we have for $x>15$

$$
\sum_{x<m \leq 2 x} u_{z}(m) \leq \frac{x}{\log x}+\frac{x}{\log (x / z) \log z},
$$

and using the inequality $z<\sqrt{2 x}$ we obtain for $x>200$

$$
\sum_{x<m \leq 2 x} u_{z}(m) \leq \frac{x}{\log z}\left(\frac{\log \sqrt{2 x}}{\log x}+\frac{1}{\log \sqrt{x / 2}}\right) \leq \frac{x}{\log z}
$$

and it suffices to show the result for $x \leq 200$ and $z<\sqrt{2 x}$, which can be verified easily by computer. This completes the proof of Lemma 7

Corollary 1. For $2 \leq z \leq x$ we have

$$
\sum_{x \leq m<2 x} u_{z}(m) \leq \frac{x}{\log z} .
$$

Proof. If $x$ is not an integer or if $x$ is an integer and $z=x$ (in this case $u_{z}(x)=0$ ), we have

$$
\sum_{x \leq d<2 x} u_{z}(d) \leq \sum_{x<d \leq 2 x} u_{z}(d) \leq \frac{x}{\log z} .
$$

If $x$ is an integer and $z<x$, we have

$$
\sum_{x \leq d<2 x} u_{z}(d)=\sum_{x^{-}<d \leq 2 x^{-}} u_{z}(d) \leq \frac{x}{\log z} .
$$

Lemma 8. For $x \geq 2$ and $1 \leq h \leq x$ we have

$$
\sum_{n \leq x} \Lambda(n) \Lambda(n+h) \leq 15 x(\log \log x+0.5)
$$

Proof. If $h$ is odd, we have $\Lambda(n) \Lambda(n+h)=0$ if $n$ is not a power of 2 . Hence, when $h$ is odd,

$$
\sum_{n \leq x} \Lambda(n) \Lambda(n+h) \leq \sum_{r \leq \frac{\log x}{\log 2}} \log 2 \log 2 x \leq \log x \log 2 x \leq 2 x .
$$

We can suppose that $h$ is even, and $\Lambda(n) \Lambda(n+h) \neq 0$ implies that $n$ is odd; therefore $n \geq 3$ and $x \geq 3$.

The contribution of the terms for which $n$ and $n+h$ are not both primes is at most

$$
2 \log 2 x \sum_{\substack{p^{r} \leq 2 x \\ r \geq 2}} \log p \leq 2 \pi(\sqrt{2 x}) \log ^{2} 2 x .
$$

By inequality 3.6 of Rosser and Schoenfeld [8] we have

$$
\forall x>1, \quad \pi(x)<1.25506 \frac{x}{\log x} ;
$$


therefore the contribution of the terms for which $n$ and $n+h$ are not both primes is at most

$$
7.1 \sqrt{x} \log 2 x .
$$

By the theorem of Siebert [10 the number of primes $p \leq x$ such that $p+h$ is prime is at most

$$
16 \prod_{p \geq 3}\left(1-\frac{1}{(p-1)^{2}}\right) \frac{x}{\log ^{2} x} \prod_{\substack{p \mid h \\ p \geq 3}} \frac{p-1}{p-2} .
$$

We remark that

$$
\frac{p-1}{p-2}=\left(1-\frac{1}{p}\right)^{-1}\left(1-\frac{1}{(p-1)^{2}}\right)^{-1},
$$

and when $h$ is even

$$
\prod_{\substack{p \backslash h \\ p \geq 3}}\left(1-\frac{1}{p}\right)=2 \frac{\varphi(h)}{h}
$$

so that Siebert's expression can be written as

$$
8 \prod_{\substack{p \\(p, h)=1}}\left(1-\frac{1}{(p-1)^{2}}\right) \frac{x}{\log ^{2} x} \cdot \frac{h}{\varphi(h)} \leq \frac{8 x}{\log ^{2} x} \cdot \frac{h}{\varphi(h)} .
$$

By inequality 3.41 and 3.42 of Rosser and Schoenfeld [8] we have for $h \geq 3$

$$
\frac{h}{\varphi(h)} \leq e^{\gamma} \log \log h+\frac{2.50637}{\log \log h}
$$

hence for $x \geq 3$ we have

$$
\sum_{n \leq x} \Lambda(n) \Lambda(n+h) \leq 8 \frac{\log 2 x}{\log x} x\left(e^{\gamma} \log \log x+\frac{2.50637}{\log \log x}\right)+7.1 \sqrt{x} \log 2 x,
$$

and for $x \geq 10^{8}$ we obtain

$$
\sum_{n \leq x} \Lambda(n) \Lambda(n+h) \leq 15 x(\log \log x+0.5) .
$$

For $x<10^{8}$ we have

$$
\sum_{n \leq x} \Lambda(n) \Lambda(n+h) \leq \log 2 x \sum_{n \leq x} \Lambda(n),
$$

and we use the inequality 3.35 of Rosser and Schoenfeld 8

$$
\sum_{n \leq x} \Lambda(n)<1.03883 x \text { for all } x>0,
$$

which gives for $10 \leq x<10^{8}$

$$
\sum_{n \leq x} \Lambda(n) \Lambda(n+h) \leq 1.03883 \log \left(2.10^{8}\right) x \leq 20 x \leq 15 x(\log \log x+0.5) .
$$

For $x<10$ the inequality is verified by direct computation. 


\section{Sums OF TYPE I AND II}

For $z \geq 3$ depending on $x$ only we can split $S(x, \alpha)$ as follows:

$$
S(x, \alpha)=\sum_{n \leq x} \Lambda(n) \mathrm{e}(n \alpha)=S_{1}(x, \alpha)+S_{2}(x, \alpha),
$$

where

$$
\begin{aligned}
& S_{1}(x, \alpha)=\sum_{n \leq x} v_{z}(n) \Lambda(n) \mathrm{e}(n \alpha), \\
& S_{2}(x, \alpha)=\sum_{n \leq x} u_{z}(n) \Lambda(n) \mathrm{e}(n \alpha) .
\end{aligned}
$$

We can estimate $S_{1}(x, \alpha)$ trivially:

$$
\left|S_{1}(x, \alpha)\right| \leq \sum_{\substack{p^{r} \leq x \\ p \leq z}} \log p=\sum_{p \leq z} \log p\left\lfloor\frac{\log x}{\log p}\right\rfloor \leq \pi(z) \log x \leq z \log x .
$$

Now we split $S_{2}(x, \alpha)$ into $B_{1}(x, \alpha)-B_{2}(x, \alpha)$ (see [4] for details) where

$$
\begin{aligned}
B_{1}(x, \alpha) & =\sum_{n \leq x} u_{z}(n) \log (n) \mathrm{e}(n \alpha), \\
B_{2}(x, \alpha) & =\sum_{z \leq d \leq x / z} u_{z}(d) \sum_{z \leq m \leq x / d} u_{z}(m) \Lambda(m) \mathrm{e}(m d \alpha) .
\end{aligned}
$$

6. Sums of type I

Lemma 9. For $\alpha=\frac{a}{q}+\frac{\beta}{q^{2}},|\beta| \leq 1,(a, q)=1,3^{7} \leq z^{7} \leq y \leq x$, we have

$$
\begin{aligned}
\left|B_{1}(x, \alpha)\right| \leq & \frac{2}{3} e^{\gamma} x \log x \log 3 z \exp \left(-\frac{\log y}{\log z}\right) \\
& +2 \frac{x}{q} \log x \log 3 y+\frac{2}{\pi} y \log x \log 4 q+\frac{3}{\pi} q \log x \log 5 q .
\end{aligned}
$$

Proof. We write

$$
B_{1}(x, \alpha)=\sum_{n \leq x} u_{z}(n) \mathrm{e}(n \alpha) \int_{1}^{n} \frac{d t}{t}=\int_{1}^{x} \frac{d t}{t} \sum_{t \leq n \leq x} u_{z}(n) \mathrm{e}(n \alpha) .
$$

Introducing $T_{1}(t, x, \alpha)=\sum_{t \leq n \leq x} u_{z}(n) \mathrm{e}(n \alpha)$, we see that

$$
\left|B_{1}(x, \alpha)\right| \leq \log x \sup _{1 \leq t \leq x}\left|T_{1}(t, x, \alpha)\right| .
$$

By the Möbius inversion formula

$$
T_{1}(t, x, \alpha)=\sum_{\substack{n, d \\ t \leq n d \leq x}} v_{z}(n) \mu(n) \mathrm{e}(n d \alpha) .
$$

Let $y$ such that $z^{7} \leq y \leq x$. We have

$$
\begin{aligned}
T_{1,1}(t, x, \alpha) & =\sum_{n \leq y} \sum_{t \leq n d \leq x} v_{z}(n) \mu(n) \mathrm{e}(n d \alpha), \\
T_{1,2}(t, x, \alpha) & =\sum_{y<n \leq x} \sum_{t \leq n d \leq x} v_{z}(n) \mu(n) \mathrm{e}(n d \alpha) .
\end{aligned}
$$


Clearly

$$
\left|T_{1,1}(t, x, \alpha)\right| \leq \sum_{n \leq y} \min \left(\frac{x}{n}, \frac{1}{|\sin (\pi n \alpha)|}\right) .
$$

Hence by Lemma 3

$$
\left|T_{1,1}(t, x, \alpha)\right| \leq 2 \frac{x}{q} \log 3 y+\frac{2}{\pi} y \log 4 q+\frac{3}{\pi} q \log 5 q .
$$

We have

$$
\left|T_{1,2}(t, x, \alpha)\right| \leq \sum_{n>y} v_{z}(n)|\mu(n)| \sum_{d \leq x / n} 1 \leq x \sum_{n>y} \frac{v_{z}(n)}{n} \mu^{2}(n) .
$$

In [8, Rosser and Schoenfeld proved (inequality 3.24) that

$$
\sum_{p \leq x} \frac{\log p}{p}<\log x \text { for all } x>1 .
$$

Using this inequality we get

$$
0<S=\sum_{p \leq z} \frac{\frac{1}{p}}{1+\frac{1}{p}} \log p \leq \sum_{p \leq z} \frac{\log p}{p} \leq \log z \leq \frac{\log y}{7} .
$$

Hence by Rankin's method (Lemma 4) we get

$$
\left|T_{1,2}(t, x, \alpha)\right| \leq x\left(\prod_{p \leq z}\left(1+\frac{1}{p}\right)\right) \exp \left(-\frac{\log y}{\log z}\right) .
$$

Now for $z \geq 3$ we have

$$
\prod_{p \leq z}\left(1+\frac{1}{p}\right) \prod_{p \leq z}\left(1-\frac{1}{p}\right)=\prod_{p \leq z}\left(1-\frac{1}{p^{2}}\right) \leq \frac{3}{4} \cdot \frac{8}{9}=\frac{2}{3} .
$$

In [8], Rosser and Schoenfeld proved (inequality 3.31) that

$$
\prod_{p \leq x} \frac{p}{p-1}<e^{\gamma} \sum_{1 \leq n \leq x} \frac{1}{n} \quad \text { for all } x \geq 1 .
$$

Using these inequalities we obtain

$$
\left|T_{1,2}(t, x, \alpha)\right| \leq \frac{2}{3} e^{\gamma} x\left(\sum_{1 \leq n \leq z} \frac{1}{n}\right) \exp \left(-\frac{\log y}{\log z}\right),
$$

and finally

$$
\left|T_{1,2}(t, x, \alpha)\right| \leq \frac{2}{3} e^{\gamma} x \log 3 z \exp \left(-\frac{\log y}{\log z}\right),
$$

which completes the proof. 


\section{SuMS OF TYPE II}

Let $J$ satisfy $2^{J}\lceil z\rceil \leq x / z<2^{J+1}\lceil z\rceil$. We have

$$
\left|B_{2}(x, \alpha)\right| \leq \sum_{0 \leq j \leq J} \sum_{2^{j}\lceil z\rceil \leq d<2^{j+1}\lceil z\rceil} u_{z}(d)\left|\sum_{z \leq m \leq x / d} u_{z}(m) \Lambda(m) \mathrm{e}(m d \alpha)\right| .
$$

We observe that $J \log 2 \leq \log x-2 \log z \leq \log x-\log 2$, and we define

$$
T_{2}(M)=\sum_{M \leq d<2 M} u_{z}(d)\left|\sum_{z \leq m \leq x / d} u_{z}(m) \Lambda(m) \mathrm{e}(m d \alpha)\right| .
$$

We get

$$
\left|B_{2}(x, \alpha)\right| \leq \frac{\log x}{\log 2} \sup _{\substack{z \leq M \leq x / z \\ M \in \mathbb{N}}}\left|T_{2}(M)\right| .
$$

By the Cauchy-Schwarz inequality

$$
\left|T_{2}(M)\right|^{2} \leq\left(\sum_{M \leq d<2 M} u_{z}^{2}(d)\right) \sum_{M \leq d<2 M}\left|\sum_{z \leq m \leq x / d} u_{z}(m) \Lambda(m) \mathrm{e}(m d \alpha)\right|^{2} .
$$

By Corollary 1, we have

$$
\sum_{M \leq d<2 M} u_{z}^{2}(d) \leq \frac{M}{\log z}
$$

Expanding the square and summing first over the $d$ 's we obtain

$$
\left|T_{2}(M)\right|^{2} \leq \frac{M}{\log z} \sum_{z \leq m \leq x / M} \Lambda(m) \sum_{z \leq m^{\prime} \leq x / M} \Lambda\left(m^{\prime}\right)\left|\sum_{d \in I\left(m, m^{\prime}\right)} \mathrm{e}\left(\left(m-m^{\prime}\right) d \alpha\right)\right|,
$$

where $I\left(m, m^{\prime}\right)$ is the interval of $d^{\prime}$ 's such that $M \leq d \leq \min \left(2 M-1, \frac{x}{m}, \frac{x}{m^{\prime}}\right)$.

We distinguish $m=m^{\prime}$ and $m \neq m^{\prime}$ and obtain

$$
\left|T_{2}(M)\right|^{2} \leq\left|T_{2,1}(M)\right|^{2}+\left|T_{2,2}(M)\right|^{2},
$$

where

$$
\left|T_{2,1}(M)\right|^{2}=\frac{M^{2}}{\log z} \sum_{z \leq m \leq x / M} \Lambda^{2}(m)
$$

and

$$
\left|T_{2,2}(M)\right|^{2}=2 \frac{M}{\log z} \sum_{1 \leq h \leq x / M} \sum_{z \leq m \leq x / M} \Lambda(m) \Lambda(m+h)\left|\sum_{d \in I(m, m+h)} \mathrm{e}(h d \alpha)\right| .
$$

We have

$$
\left|\sum_{d \in I(m, m+h)} \mathrm{e}(h d \alpha)\right| \leq \min \left(M, \frac{1}{|\sin (\pi h \alpha)|}\right) .
$$


By Lemma 8

$$
\sum_{z \leq m \leq x / M} \Lambda(m) \Lambda(m+h) \leq 15(\log \log x+0.5) \frac{x}{M} .
$$

So

$$
\left|T_{2,2}(M)\right|^{2} \leq 30(\log \log x+0.5) \frac{x}{\log z} \sum_{1 \leq h \leq x / M} \min \left(M, \frac{1}{|\sin (\pi h \alpha)|}\right) .
$$

Using Lemma 2 and $z \leq M \leq x / z$ we get

$$
\begin{aligned}
\sum_{1 \leq h \leq x / M} \min \left(M, \frac{1}{|\sin (\pi h \alpha)|}\right) & \leq\left(\frac{x}{M q}+1\right)\left(2 M+\frac{2}{\pi} q \log 4 q\right) \\
& \leq \frac{2 x}{q}+2 M+\frac{2 x \log 4 q}{\pi M}+\frac{2}{\pi} q \log 4 q \\
& \leq \frac{2 x}{q}+\frac{2 x}{z}+\frac{2 x \log 4 q}{\pi z}+\frac{2}{\pi} q \log 4 q \\
& \leq 2 x\left(\frac{1}{q}+\frac{\pi+\log 4 q}{\pi z}+\frac{q \log 4 q}{\pi x}\right) .
\end{aligned}
$$

We obtain

$$
\begin{aligned}
\left|T_{2,2}(M)\right|^{2} \leq & 60(\log \log x+0.5) \frac{x^{2}}{\log z}\left(\frac{1}{q}+\frac{\log 93 q}{\pi z}+\frac{q \log 4 q}{\pi x}\right), \\
& \sum_{n \leq x} \Lambda(n)<1.03883 x \quad \text { for all } x>0 .
\end{aligned}
$$

For $z \geq 3$, the function $M \longmapsto M \log (x / M)$ is increasing on $[z, x / z]$. Hence by Rosser and Schoenfeld [8] inequality 3.35 we have

$$
\left|T_{2,1}(M)\right|^{2} \leq 1.03883 x M \frac{\log (x / M)}{\log z} \leq 1.03883 \frac{x^{2}}{z}
$$

and

$$
\begin{aligned}
\left|B_{2}(x, \alpha)\right| \leq & \frac{\sqrt{1.03883}}{\log 2} \frac{x}{\sqrt{z}} \log x \\
& +\frac{1}{\log 2} \sqrt{60(\log \log x+0.5)} \frac{x \log x}{\sqrt{\log z}}\left(\sqrt{\frac{1}{q}+\frac{q \log 4 q}{\pi x}}+\sqrt{\frac{\log 93 q}{\pi z}}\right) .
\end{aligned}
$$

Finally

$$
\begin{aligned}
\left|B_{2}(x, \alpha)\right| \leq & 1.48 \frac{x}{\sqrt{z}} \log x \\
& +11.18 \sqrt{\log \log x+0.5} \frac{x \log x}{\sqrt{\log z}}\left(\sqrt{\frac{1}{q}+\frac{q \log 4 q}{\pi x}}+\sqrt{\frac{\log 93 q}{\pi z}}\right) .
\end{aligned}
$$




\section{Proof of Theorem 1}

We can suppose $x \geq 10^{184}$; otherwise

$$
\sqrt{\log \log x+0.5} x(\log x)^{5 / 4} \exp \left(-\frac{1}{2} \sqrt{\log x}\right)>0.166
$$

and the result is trivial using Rosser and Schoenfeld [8] inequality 3.35. Furthermore we can suppose

$$
(\log x)^{3 / 2} \log \log x \leq q \leq \frac{x}{(\log x)^{5 / 2} \log \log x}
$$

otherwise the result is trivial.

We choose $\log z=\sqrt{\log x}$ and we obtain

$$
\begin{aligned}
\left|B_{2}(x, \alpha)\right| \leq & 11.18 \sqrt{\log \log x+0.5} x(\log x)^{3 / 4} \sqrt{\frac{1}{q}+\frac{q \log 4 q}{\pi x}} \\
& +1.48 x \log x \exp \left(-\frac{1}{2} \sqrt{\log x}\right) \\
& +6.31 \sqrt{\log \log x+0.5} x(\log x)^{5 / 4} \exp \left(-\frac{1}{2} \sqrt{\log x}\right) \\
\leq & 11.18 \sqrt{\log \log x+0.5} x(\log x)^{3 / 4} \sqrt{\frac{1}{q}+\frac{q \log 4 q}{\pi x}} \\
& +6.44 \sqrt{\log \log x+0.5} x(\log x)^{5 / 4} \exp \left(-\frac{1}{2} \sqrt{\log x}\right)
\end{aligned}
$$

Let us first suppose that

$$
(\log x)^{3 / 2} \log \log x \leq q \leq(\log x)^{3}
$$

Let $\log y=\sqrt{\log x} \log q$. We then have for $x \geq 10^{184}$

$$
\begin{aligned}
\left|B_{1}(x, \alpha)\right| \leq & 1.19 \frac{x}{q} \log x(\log z+\log 3)+2 \frac{x}{q} \log x(\log y+\log 3) \\
& +0.64 \exp (\sqrt{\log x} \log q) \log x \log 4 q+0.96 q \log x \log 5 q \\
\leq & 3.68 \sqrt{\log \log x+0.5} x(\log x)^{3 / 4} \sqrt{\frac{1}{q}} .
\end{aligned}
$$

Now let us suppose that

$$
(\log x)^{3}<q \leq \frac{x}{(\log x)^{5 / 2} \log \log x}
$$

We choose

$$
y=x(\log x)^{-1 / 2} \exp (-\sqrt{\log x}),
$$




$$
\begin{aligned}
\left|B_{1}(x, \alpha)\right| \leq & 3.95 x(\log x)^{3 / 2} \exp (-\sqrt{\log x})+2 \frac{x}{q}(\log x)^{2} \\
& +\frac{2}{\pi} x(\log x)^{3 / 2} \exp (-\sqrt{\log x})+\frac{3}{\pi} q \log x \log 5 q \\
\leq & 5.59 x(\log x)^{3 / 2} \exp (-\sqrt{\log x})+0.45 x(\log x)^{3 / 4} \sqrt{\frac{1}{q}} \\
& +0.11 x(\log x)^{3 / 4} \sqrt{\frac{q \log 4 q}{\pi x}} \\
\leq & 0.56 x(\log x)^{3 / 4} \sqrt{\frac{1}{q}+\frac{2}{3 \pi} \cdot \frac{q \log 4 q}{x}} \\
& +4.59 x(\log x)^{3 / 2} \exp (-\sqrt{\log x}) .
\end{aligned}
$$

Hence for all $q$ we have

$$
\begin{aligned}
\left|B_{1}(x, \alpha)\right| \leq & 3.68 \sqrt{\log \log x+0.5} x(\log x)^{3 / 4} \sqrt{\frac{1}{q}+\frac{2}{3 \pi} \cdot \frac{q \log 4 q}{x}} \\
& +0.01 \sqrt{\log \log x+0.5} x(\log x)^{5 / 4} \exp \left(-\frac{1}{2} \sqrt{\log x}\right) .
\end{aligned}
$$

Finally we have

$$
\begin{aligned}
|S(x, \alpha)| \leq & \left|S_{1}(x, \alpha)\right|+\left|B_{1}(x, \alpha)\right|+\left|B_{2}(x, \alpha)\right| \\
\leq & 14.86 \sqrt{\log \log x+0.5} x(\log x)^{3 / 4} \sqrt{\frac{1}{q}+\frac{2}{3 \pi} \cdot \frac{q \log 4 q}{x}} \\
& +6.45 \sqrt{\log \log x+0.5} x(\log x)^{5 / 4} \exp \left(-\frac{1}{2} \sqrt{\log x}\right) .
\end{aligned}
$$

\section{REFERENCES}

[1] J. Chen, On the estimation of some trigonometrical sums and their application (Chinese), Scientia Sinica, 28 (1985), pp. 449-458. MR 87h:11078

[2] J. Chen And T. Wang, On the Goldbach problem (Chinese), Acta Mathematica Sinica, 32 (1989), No. 5, pp. 702-718. MR 91e:11108

[3] Estimation of linear trigonometric sums with primes (Chinese), Acta Mathematica Sinica, 37 (1994), No. 1, pp. 25-31. MR 95c:11102

[4] H. Daboussi, Effective estimates of exponential sums over primes, Analytic Number Theory, Vol. 1, Progr. Math., 138, Birkhäuser, Boston, 1996, pp. 231-244. MR 97i:11088

[5] P. Dusart, Autour de la fonction qui compte le nombre de nombres premiers, PhD thesis, Université de Limoges, 1998.

[6] P. D. T. A. Elliott, Probabilistic Number Theory I, vol. 239 of Grundlehren der mathematichen Wissenschaften, Springer-Verlag, 1979. MR 82h:10002a

[7] H. L. Montgomery and R. C. Vaughan, The large sieve, Mathematika, 20 (1973), pp. 119134. MR 51:10260

[8] J. B. Rosser and L. Schoenfeld, Approximate formulas for some functions of prime numbers, Illinois Journal of Mathematics, 6 (1962), pp. 64-94. MR 25:1139

[9] Sharper bounds for the Chebyshev functions $\theta(x)$ and $\psi(x)$, Mathematics of Computation, 29 (1975), pp. 243-269. MR 56:15581a

[10] H. Siebert, Montgomery's weighted sieve for dimension two, Monatshefte für Mathematik, 82 (1976), pp. 327-336. MR 54:12690

[11] R. C. Vaughan, An elementary method in prime number theory, Acta Arithmetica, 37 (1980), pp. 111-115. MR 82c:10055

[12] I. M. Vinogradov, Representation of an odd number as the sum of three primes, Dokl. Akad. Nauk SSSR, 15 (1937), pp. 291-294. 
[13] The method of Trigonometrical Sums in the Theory of Numbers, translated from the Russian, revised and annotated by K.F. Roth and A. Davenport, Interscience, London, 1954. MR 15:941b

Faculté de Mathématiques et D'Informatique, 33 Rue Saint-Leu, 80039 Amiens, France Current address: UMR CNRS 8752, Mathématiques, Université Paris-Sud, 91405 Orsay Cedex, France

E-mail address: daboussi@math.u-psud.fr

Institut Girard Desargues, CNRS UPRES-A 5028, Université Lyon I, 43, Boulevard Du 11 novembre 1918, 69622 Villeurbanne Cedex, France

Current address: Institut Elie Cartan, Université Henri Poincaré, B.P. 239, 54506 Vandoeuvre cedex, France

E-mail address: rivat@iecn.u-nancy.fr 